\title{
Chromatographic behavior of epirubicin and its analogues on high-purity silica in hydrophilic interaction chromatography
}

\author{
Ruiping Li, Junxiong Huang* \\ Research Center for Eco-Environmental Sciences, Chinese Academy of Sciences, P.O. Box 2871, Beijing 100085, China
}

Received 16 January 2004; received in revised form 22 March 2004; accepted 26 April 2004

Available online 25 May 2004

\begin{abstract}
Hydrophilic interaction chromatography has been applied for the separation of epirubicin and its analogues using high-purity silica column with aqueous-organic mobile phase. Parameters affecting the chromatographic behavior of the solutes such as organic modifier, buffer $\mathrm{pH}$, ionic strength and sample size, have been investigated. Of utmost importance for successful separation of these analogues is the choice of organic modifier, since it impacts both the solvent selectivity and the ionization of silica silanols as well as buffer solution, and consequently the retention behavior of solutes. Acetonitrile was shown to offer superior separation of these analogues to methanol, isopropanol or tetrahydrofuran. Results of the effects of organic modifier, buffer $\mathrm{pH}$ and ion strength indicate that the retention mechanism is a mixed-mode of adsorption and ion exchange. In addition, an irreversible adsorption of these compounds was found on silica in the weakly acidic or neutral mobile phases, and the effect of various factors on irreversible adsorption was also preliminarily discussed. More significantly, these basic compounds have exhibited peaks with a slanted front and a sharp tail, a typical overloading peak profile belonging to the behavior of competitive anti-Langmuir isotherm by increasing the sample size at the experimental conditions.
\end{abstract}

(C) 2004 Elsevier B.V. All rights reserved.

Keywords: Hydrophilic interaction chromatography; Mobile phase composition; Adsorption isotherms; Epirubicin; Antibiotics

\section{Introduction}

Reversed-phase liquid chromatography (RPLC) has become a widely used separation method in the modern chromatographic science. After several decades of development, silica-based stationary phases remain the first choice for most RPLC separations [1], however, it is not always the best one for the separation of some polar compounds, since these compounds often show inadequate retention or broad tailing peaks $[2,3]$. For some polar samples, another useful, effective mode of chromatography, i.e. hydrophilic interaction chromatography (HILIC) often works well where RPLC is ineffective [4].

HILIC termed by Alpert in 1990 is able to separate compounds by passing aqueous-organic mobile phases across a polar stationary phase such as silica, diol phase and otherwise, causing solutes to elute in order of increasing

\footnotetext{
* Corresponding author. Tel.: +86-10-6294-3703; fax: +86-10-6284-9337.

E-mail address: junxionghuang@yahoo.com (J. Huang).
}

hydrophilicity-the opposite of RPLC [5]. It is a variant of normal-phase liquid chromatography (NPLC) in which water is the strongest solvent. Applications have emerged in the separation of amino acids and peptides [5,6], carbohydrates [7], polar glycopeptide antibiotics [8,9], pyrimidines and other polar pharmaceuticals [4], etc. with various polar columns.

Silica with aqueous-organic mobile phases has been successfully used for basic drugs analysis under HILIC conditions [4,10-12]. The advantages of this chromatographic system include high separation efficiency, good peak shape, short retention time, simple mobile phases (e.g. no ion-pair or ion suppression reagents, etc.), low column cost, and a possibility to enhance retention for some species (e.g. protonated amines) [4,10-12]. Moreover, silica is especially useful for separating difficult mixtures containing geometrical isomers in NPLC mode. HILIC on silica can provide an alternative to control the selectivity and retention by changing the mobile phase compositions. In contrast with the extensive literature on the studies of the influence of $\mathrm{pH}$, ionic strength and origin of the silica [12], little work has been re- 


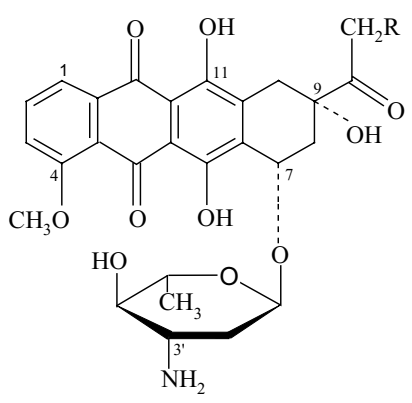

(A)

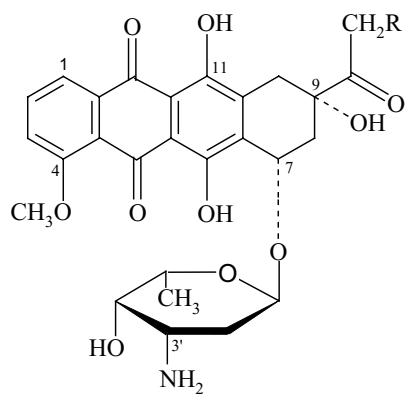

(B)
Fig. 1. Structural formulae of epirubicin and its analogues. (A) $\mathrm{R}=-\mathrm{OH}$, epirubicin ( $\left.\mathrm{p} K_{\mathrm{a}}=8.08\right) ; R=-\mathrm{H}$, epidaunorubicin ( $\left.\mathrm{p} K_{\mathrm{a}}=8.2\right) ;(\mathrm{B}) \mathrm{R}$ $=-\mathrm{OH}$, doxorubicin $\left(\mathrm{p} K_{\mathrm{a}}=8.34\right) ; \mathrm{R}=-\mathrm{H}$, daunorubicin $\left(\mathrm{p} K_{\mathrm{a}}=8.46\right)$.

ported about studies on how the selectivity of the separation of basic compounds is influenced by the nature of organic modifier, and on sample loading behavior of ionized basic solutes in this chromatographic mode.

Epirubicin (4'-epidoxorubicin), a clinically important antitumor antibiotic, has been widely used for the treatment of a variety of tumors [13]. Its related impurities are mainly doxorubicin, daunorubicin and epidaunorubicin. Both epirubicin and its impurities are weak bases and possess highly similar structures [14], for example, epirubicin and doxorubicin, differ only in $-\mathrm{OH}$ group position in the sugar fragment. The molecular structures and the $\mathrm{p} K_{\mathrm{a}}$ values for amino groups in the molecule [14] are shown in Fig. 1. Several RPLC methods have been developed for separation of epirubicin and related compounds [15-20]. In our previous work, milligram amounts of epirubicin were isolated from raw product by RPLC carried out in the overloaded elution mode [21] and in the displacement mode [22] on an analytical-scale Kromasil KR100-10C 18 column. The purpose of this study is to explore the feasibility and utility of HILIC for the separation of epirubicin from its impurities on high-purity silica, to investigate the impacts of various experimental parameters, such as organic modifier and its concentration, buffer $\mathrm{pH}$, ionic strength and sample size on the chromatographic behavior of these analogues, and to discuss preliminarily the retention mechanism using a mixed-mode and other phenomena occurred in chromatographic process. Of special interest is the effect of organic modifier nature and sample size, which will not only further provide some insights of the retention mechanism of these basic drugs, but also imply additional information about the properties of high-purity silica in aqueous-organic mobile phases.

\section{Experimental}

\subsection{Apparatus and reagents}

The HPLC system from a TSP (Thermo Separation Products, San Jose, CA, USA) consisted of a SpectraSystem
P4000 pump, a SpectraSystem AS3000 autosampler with a fixed-loop injection valve, and a Spectra FOCUS diode array detector. Chromatographic system control, data acquisition, and chromatographic analysis were exerted with TSP PC1000 Chromatography Manager software (3.0 version). The column used was Kromasil KR100-5SIL, $250 \mathrm{~mm} \times$ $4.6 \mathrm{~mm}$ i.d., pore size $100 \AA$, particle size $5 \mu \mathrm{m}$, specific surface area $330 \mathrm{~m}^{2} / \mathrm{g}$ (Eka, Bohus, Sweden). Acetonitrile and methanol were of HPLC grade (Tedia, USA). All other solvents and reagents were of analytical-reagent grade. Water was purified by a Milli-Q system (Millipore, Bedford, MA, USA). Epirubicin, doxorubicin, daunorubicin, epidaunorubicin standards and epirubicin raw material were obtained as a gift from Hisun Pharmaceutical (Zhejiang, China). All reagents were used as received without further purification.

\subsection{Chromatographic conditions}

All separations were operated at ambient temperature. The mobile phases were pump-mixed dynamically from formate buffers and acetonitrile at specified compositions. Note that buffer concentrations and $\mathrm{pH}$ values refer to the aqueous portion alone. The buffers were prepared from sodium formate, adjusting to the required $\mathrm{pH}$ with formic acid $(\sim 88 \%$, $\mathrm{v} / \mathrm{v})$. Solutes were detected at UV $254 \mathrm{~nm}$. The flow rate was $1.0 \mathrm{ml} / \mathrm{min}$ and the injection volume was $20 \mu \mathrm{l}$.

\section{Results and discussion}

\subsection{Effect of mobile phase on chromatographic behavior of the sample}

\subsubsection{The nature of organic modifier}

The C-3' amino group and the phenolic hydroxyls in epirubicin and its analogues can be present in differently charged forms in aqueous media, and causing different chromatographic behavior. At $\mathrm{pH}$ below 7, the protonated form is the only ionic species present in their aqueous solution and they will behave as a zwitterion or an anion at higher $\mathrm{pH}$ values [14]. In order to examine the influence of organic modifier on the separation of epirubicin and its analogues on silica, the acidic buffer was selected to keep both the solutes and the majority of silanol groups on the silica surface in protonated forms. In this study, the mixture of the four compounds was chromatographed using formate buffer $(20 \mathrm{mM}$, $\mathrm{pH}$ 2.9) combined individually with methanol, acetonitrile, isopropanol and tetrahydrofuran (THF) at the same concentration (10:90, v/v) as mobile phases. A typical comparison of chromatograms obtained is shown in Fig. 2. It indicates that, modifier strength for eluting the four analogues has been increased in following series: acetonitrile $<\mathrm{THF}<$ isopropanol $<$ methanol, which is partly inconsistent with the eluotropic series of these solvents [23]. Among the four organic modifiers tested, methanol caused all four solutes to be eluted with no retention. Acetonitrile provides a best res- 


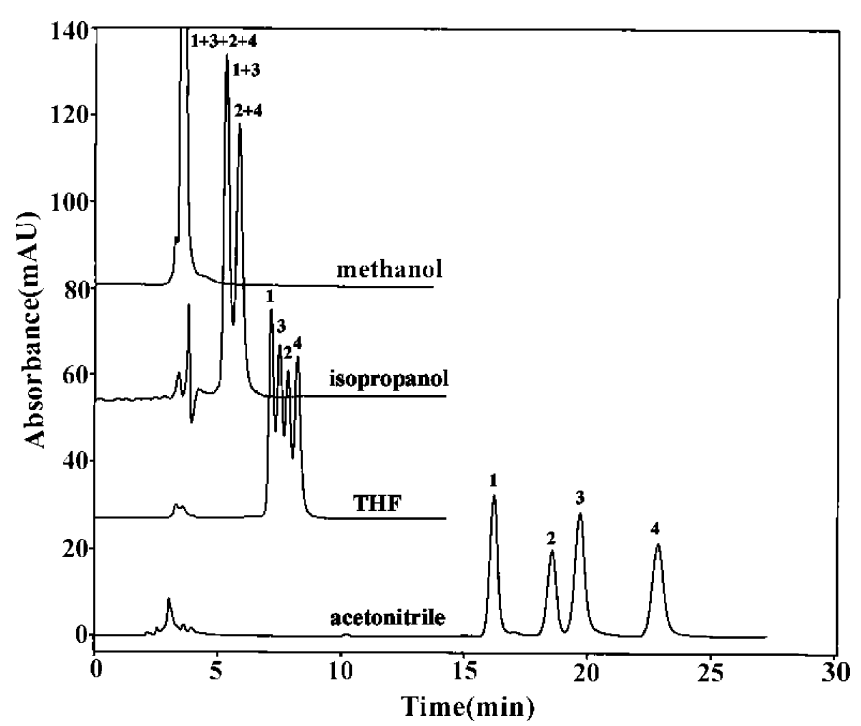

Fig. 2. Effect of organic modifier on separation of epirubicin and its analogues. Conditions: Kromasil KR100-5SIL ( $5 \mu \mathrm{m})$; mobile phase: sodium formate buffer $(20 \mathrm{mM}, \mathrm{pH} 2.9)$ modified with various organic solvents (10:90, v/v). Peaks: (1) epidaunorubicin, (2) daunorubicin, (3) epirubicin, (4) doxorubicin.

olution of the mixture, and the elution order of the solutes in this case is just opposite to that in RPLC [21]. Meanwhile, the peaks elution order with acetonitrile is different from that with THF.

The above observations indicate that there are competitive adsorptions between the solutes and the polar organic modifier. Moreover, the influences of organic modifier on the dissociation of buffer compound and the $\mathrm{p} K_{\mathrm{a}}$ of both analytes and silanol groups of silica have played a role in the resolution of solutes $[24,25]$. The combination of these effects makes it rather difficult to unravel our experimental results with common theoretical models in HPLC. In our case, the components of the mobile phase are localizing solvents. Water just deactivates highly adsorptive centers and has no further effect on the retention process, even though it is the strongest solvent with comparatively high percentage [23]. In other words, the organic modifiers dominate the retention behavior of the solutes under the conditions where water content is fixed, with the organic modifier being variable.

According to Snyder [26], there are three major contributions to solvent selectivity, i.e. solvent strength, solvent-solute localization and solvent-solute hydrogen bonding. The solvent strengths of methanol $\left(\varepsilon^{\mathrm{o}}=0.73\right)$ and isopropanol $\left(\varepsilon^{0}=0.60\right)$ are much stronger than those for THF $\left(\varepsilon^{\mathrm{o}}=0.48\right)$ and acetonitrile $\left(\varepsilon^{\mathrm{o}}=0.50\right)$, thus the retention times of the solutes in former two modifiers are shorter than that in latter two ones. Because of its strong ability of hydrogen bonding, methanol can perturb the formation of water layers on silica surface, replacing water molecules and producing a more hydrophobic stationary phase [27]. As a consequence, the ionic solutes are poorly retained.
Compared to methanol, a decrease of the hydrophilic character of isopropanol is caused by the longer alkyl chain. So, it competes less strongly for the active sites, leaving much more time for solute molecules to be adsorbed. But, the adsorbent cannot differentiate the molecules that have identical steric factors apart from their functional groups, e.g. epirubicin and epidaunorubicin. That is to say, the $4^{\prime}-\mathrm{OH}$ in sugar moiety (i.e. steric factor) contributes much to obtain adequate selectivity rather than the $-\mathrm{OH}$ in the acetyl side chain. This is perhaps due to steric hindrance effect, resulting in hydrogen bonding interactions more readily to occur for the $-\mathrm{OH}$ in the acetyl side chain than the $4^{\prime}-\mathrm{OH}$ in sugar moiety. The hydrogen bonding interactions between solutes and solvent molecules arise in both the mobile phase and the stationary phase, significantly affecting the solutes retention and solvent selectivity.

In contrast to methanol and isopropanol, the hydrogen bond donor strengths (denoted $\alpha$ ) of acetonitrile and THF are weak, and their $\alpha$ values are 0.15 and 0.00 , respectively. The elution strengths of acetonitrile and THF are approximate, but the solutes are retained more strongly with acetonitrile than THF. This is because THF is a better hydrogen bond acceptor (denoted $\beta$ ) than acetonitrile, and the solvent-solute localization effect is correlated with $\beta$ of the solvent [26]. The higher $\beta$ of the solvent, the lower the $\mathrm{p} K_{\mathrm{a}}$ value of the basic analyte [24]. The $\beta$ values of THF and acetonitrile are 0.49 and 0.25 , respectively. Therefore, the $\mathrm{p} K_{\mathrm{a}}$ values of solutes are expected to be higher in the mobile phase with acetonitrile than with THF. The higher the $\mathrm{p} K_{\mathrm{a}}$ the stronger is the interactions with the ion-exchange site [12]. The stronger retentions of the solutes with acetonitrile mobile phase reveals that, besides adsorption, ion exchange interactions between solutes and stationary phase exist as well, i.e. the effects of organic modifier on the dissociation of buffer compound and the $\mathrm{p} K_{\mathrm{a}}$ of the silica silanols also contribute to retention properties in HILIC [24,25]. The variation in elution order of the solutes in the two solvents is a reflection of complexity of the interferences between these effects.

In order to further prove above explanation, a similar experiment has been performed with a change of buffer at $\mathrm{pH}$ from 2.9 to 6.5 in the mobile phase with methanol as modifier. It was found that all the solutes show adequate retention, and the retention factors increase in the following sequence: epirubicin $<$ epidaunorubicin $<$ doxorubicin $<$ daunorubicin. This trend is consistent with the increasing order of $\mathrm{p} K_{\mathrm{a}}$ values for amino groups of these solutes [14]. The resolution between epirubicin and epidaunorubicin, as well as doxorubicin and daunorubicin is incomplete due to the solvent-solute hydrogen bonding, while sufficient resolution is obtained between the former two and the latter two ones. The results have shown again that the ion exchange interactions obviously dominate the retention of the solutes because of the ionization of silica silanols at elevated $\mathrm{pH}$ values, causing weakness of the adsorption interactions between methanol and silica surface. 


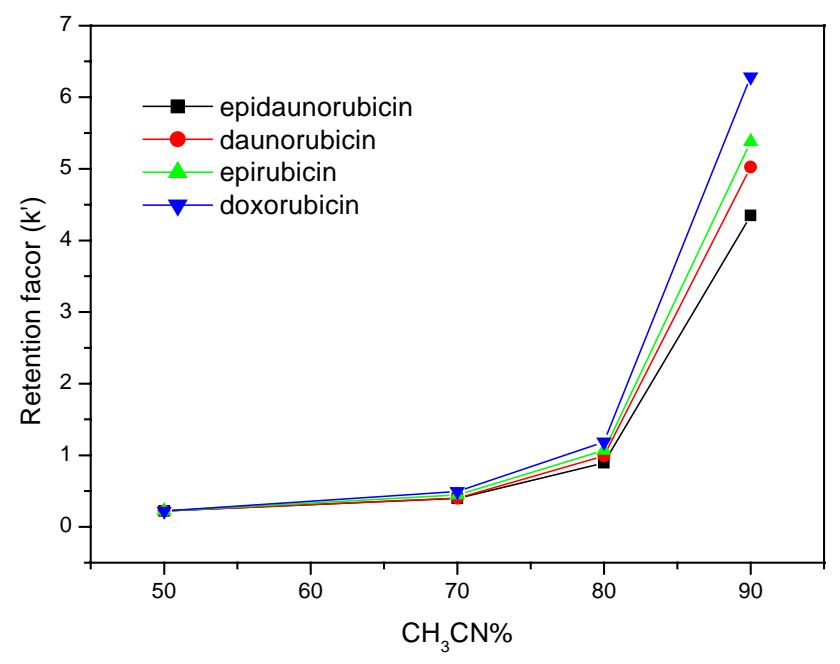

Fig. 3. Effect of acetonitrile concentration on the retention factor $\left(k^{\prime}\right)$ of epirubicin and its analogues. Conditions as given in Fig. 2, except utilizing only acetonitrile and the concentration of acetonitrile is varied.

\subsubsection{The concentration of organic modifier}

The concentration of acetonitrile was varied over the range of $50-90 \%(\mathrm{v} / \mathrm{v})$ at the buffer $\mathrm{pH}$ of 2.9 to investigate its effect on the separation of the analogues as reflected by retention factor in Fig. 3. The increase of organic modifier in mobile phase causes simultaneous increase of retention and selectivity of the solutes due to the decrease of polarity of the mobile phase, and the elution order is contrary to that obtained with RPLC [21]. With increasing acetonitrile over $70 \%$, the hydrophilic interaction chromatographic behavior of the solutes could be observed. When its concentration reaches $90 \%$, a baseline separation of the four analogues could be obtained, and further increase of the concentration of acetonitrile is unnecessary.

\subsubsection{The $p H$ of buffer solution}

Usually, high percentage of organic modifier is used under HILIC conditions [5]; thus, solubility of buffer salts is a problem with mostly organic mobile phases. In order to optimize the buffer and the selectivity, some preliminary experiments using phosphate, acetate and formate were individually tested. Ultimately, sodium formate buffer is selected to prepare the mobile phases as a compromise among reasonable solubility, available buffering capacity and sufficient resolution in this study. The effect of buffer $\mathrm{pH}$ on the separation of the four analogues is mirrored in Fig. 4 and Fig. 5.

In Fig. 4, with the buffer $\mathrm{pH}$ increasing, the retention values of all solutes have reached to a maximum and then dropped down. This behavior can be ascribed to the effects of $\mathrm{pH}$ on the ionization of surface silanols and the solutes [12]. The ionization of the silanols is gradually increased and the solutes are mainly in protonated state at $\mathrm{pH}$ range from 2.4 to 4.2 [14]. It offers the possibility of a cation-exchange mechanism. At buffer $\mathrm{pH}$ from 4.2 to 6.5 , a large percentage

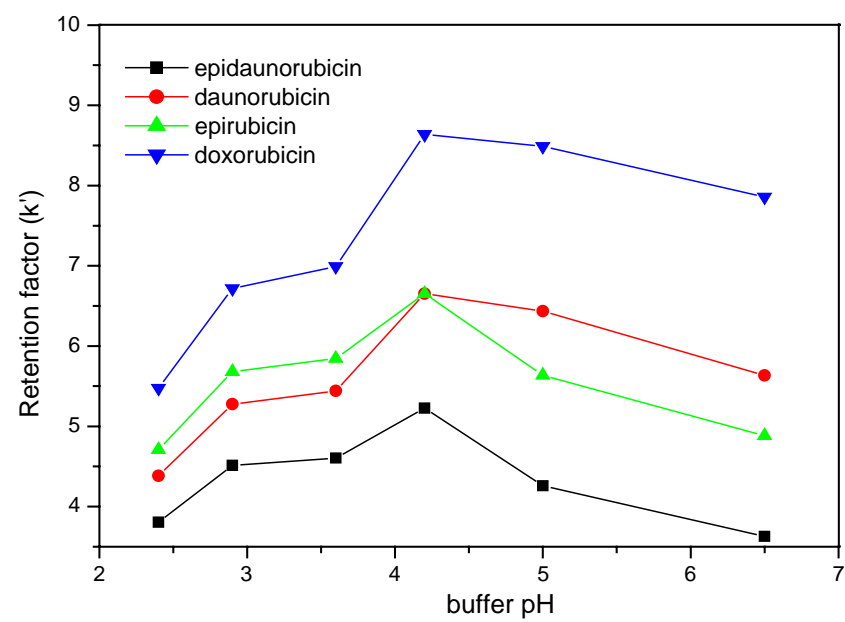

Fig. 4. Effect of buffer $\mathrm{pH}$ on the retention factor $\left(k^{\prime}\right)$ of epirubicin and its analogues. Conditions as given in Fig. 2, except utilizing only acetonitrile and the buffer $\mathrm{pH}$ is varied.

of silanols are ionized, however, the protonation of solutes is decreased. Accordingly, it reduces the ionic interaction between the solutes and ionized silanols of silica, and leads to a reduction of $k^{\prime}$ values. But, no obvious changes were noted in asymmetry factors for these compounds at $\mathrm{pH}$ values from 2.4 to 6.5 , and acceptable peak shapes are obtained (Fig. 5).

Fig. 6 displays the plots of relative retentions of the solutes to epirubicin versus buffer $\mathrm{pH}$. It can be found that the changes of $\mathrm{pH}$ from 2.4 to 3.6 have little effect on the separation selectivity for the four solutes. However, the relative retentions have been changed noticeably with increasing $\mathrm{pH}$ from 3.6 to 5.0, and to an insignificant extent from $\mathrm{pH} 5.0$ to 6.5 . Under the acidic conditions at $\mathrm{pH} 2.4-3.6$, less po-

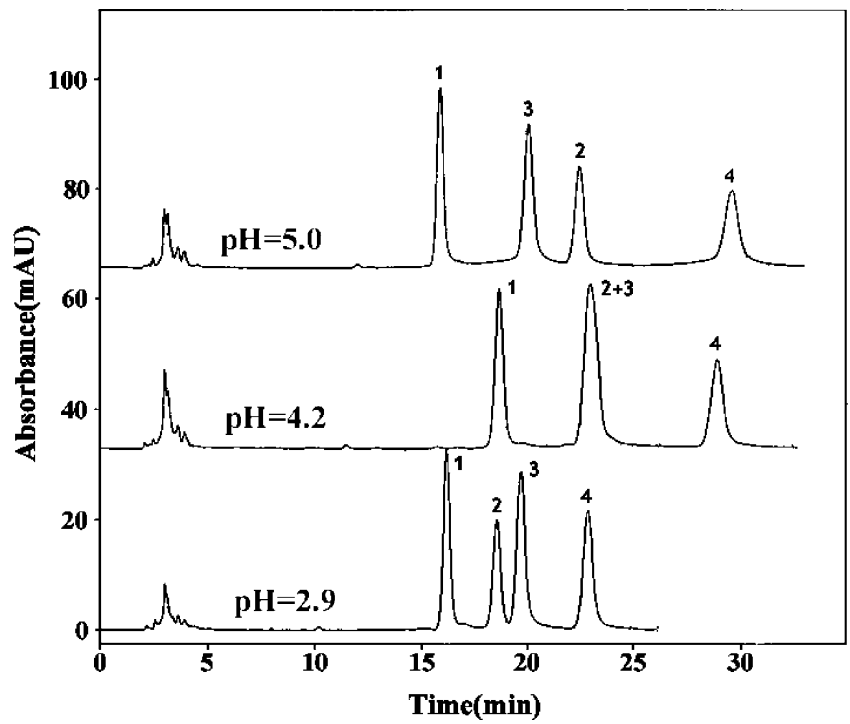

Fig. 5. Comparison of the chromatographic separation of epirubicin and its analogues at different buffer $\mathrm{pH}$. Conditions are the same as given in Fig. 4. Peaks: (1) epidaunorubicin $(0.025 \mathrm{mg} / \mathrm{ml}),(2)$ daunorubicin $(0.020 \mathrm{mg} / \mathrm{ml})$, (3) epirubicin $(0.025 \mathrm{mg} / \mathrm{ml}),(4)$ doxorubicin $(0.025 \mathrm{mg} / \mathrm{ml})$. 


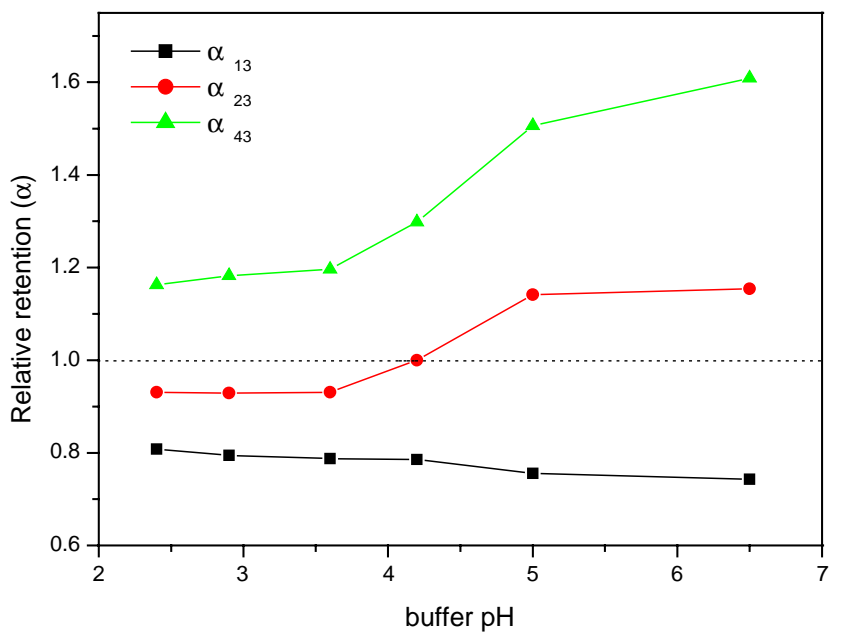

Fig. 6. Effect of buffer $\mathrm{pH}$ on the relative retention $(\alpha)$ of epirubicin and its analogues. Conditions are the same as in Fig. 4. Subscripts 1, 2, 3, 4 of $\alpha$ refers to epidaunorubicin, daunorubicin, epirubicin and doxorubicin, respectively.

lar daunorubicin eluted prior to epirubicin may reflect that adsorption mechanism predominates in separation process. With increasing the ionization of silanols at $\mathrm{pH}$ from 3.6 to 5.0, the adsorptions and ionic interactions have made a serious competition, and a coelution of the two solutes occurs at $\mathrm{pH}$ 4.2. At $\mathrm{pH}$ from 5.0 to 6.5 , daunorubicin was retained more strongly than epirubicin, reverse to the case at $\mathrm{pH}$ from 2.4 to 3.6. It indicates that ion-exchange interaction has made a major contribution to retention of solutes on silica, since the $\mathrm{p} K_{\mathrm{a}}$ value of daunorubicin $\left(\mathrm{p} K_{\mathrm{a}}=8.46\right)$ is higher than that of epirubicin $\left(\mathrm{p} K_{\mathrm{a}}=8.08\right)$, and the higher $\mathrm{p} K_{\mathrm{a}}$ of the solutes, the stronger is the interaction with the ion-exchange site [12]. This result also shows us that the separation selectivity can be adjusted according to the $\mathrm{p} K_{\mathrm{a}}$ values of the solutes and to the alteration of the $\mathrm{pH}$ value in the mobile phase. This is of particular significance for the analysis and preparative separation of this kind of analogues.

\subsubsection{The ionic strength of the mobile phase}

The influence of ionic strength of mobile phases on the retention of solutes was tested at the buffer $\mathrm{pH}$ of 2.9 and 6.5. An increase of the buffer cation strength leads to a decrease

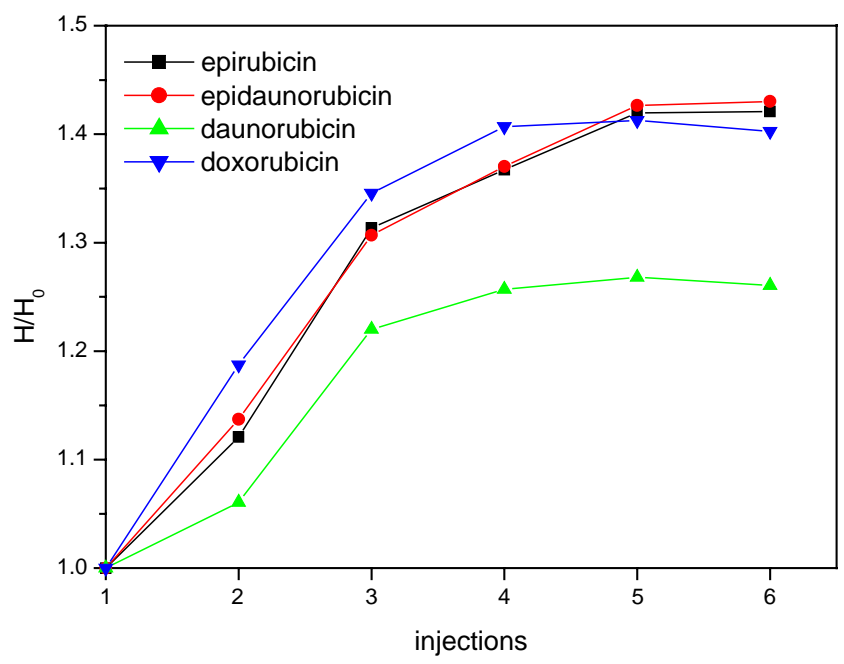

Fig. 7. Plots of $H / H_{0}$ (peak height divided by the peak height for the first injection) vs. continuous six injections. Conditions as given in Fig. 4, except the buffer $\mathrm{pH}$ of 6.5 is used.

of the retention factor for all solutes (Table 1), as a result of effective competition of the added $\mathrm{Na}^{+}$ions with solute ions for the ion exchanging sites of the stationary phase. This is an alternative proof of ion exchange mechanism playing in the process of separation in HILIC.

\subsection{Irreversible adsorption}

During the above experiments, we found that an adsorption equilibration of the solutes on the silica column was gradually reached after the column sufficiently washed with neutral or weakly acidic mobile phases, similar to the phenomenon observed previously on a $\mathrm{C}_{18}$ column in RPLC [21]. The peak heights of the components have been progressively enhanced by continuous injections from beginning and finally got to be stable (Fig. 7), accompanying with gradual improvements in resolution and column efficiency. Furthermore, with decreasing the $\mathrm{pH}$ value of the mobile phase, the column tends to need fewer repetitive injections of the sample to reach equilibrium. For instance, at the buffer $\mathrm{pH}$ of 2.4, the reproducible separations with favorable column efficiency were obtained. These observations can be

Table 1

Effect of buffer cation strength on the retention factor $\left(k^{\prime}\right)$ of epirubicin and its analogues at $\mathrm{pH} 2.9$ and 6.5

\begin{tabular}{|c|c|c|c|c|c|c|}
\hline \multirow[t]{2}{*}{$\mathrm{pH}$} & \multirow[t]{2}{*}[\mathrm{HCO}_{2}\mathrm{Na}]{$(\mathrm{mM})$} & \multirow[t]{2}{*}[\mathrm{NaNO}_{3}]{$(\mathrm{mM})$} & \multicolumn{4}{|l|}{$k^{\prime}$} \\
\hline & & & Epirubicin & Epidaunorubicin & Daunorubicin & Doxorubicin \\
\hline \multirow[t]{3}{*}{2.9} & 20 & 0 & 5.47 & 4.36 & 5.07 & 6.41 \\
\hline & 30 & 0 & 5.04 & 3.94 & 4.60 & 5.96 \\
\hline & 40 & 0 & 4.76 & 3.61 & 4.25 & 5.67 \\
\hline \multirow[t]{3}{*}{6.5} & 20 & 10 & 4.42 & 3.80 & 4.81 & 5.77 \\
\hline & 20 & 20 & 4.03 & 3.45 & 4.45 & 5.22 \\
\hline & 20 & 30 & 3.13 & 2.70 & 3.46 & 4.06 \\
\hline
\end{tabular}

Other conditions are the same as given in Fig. 5. 


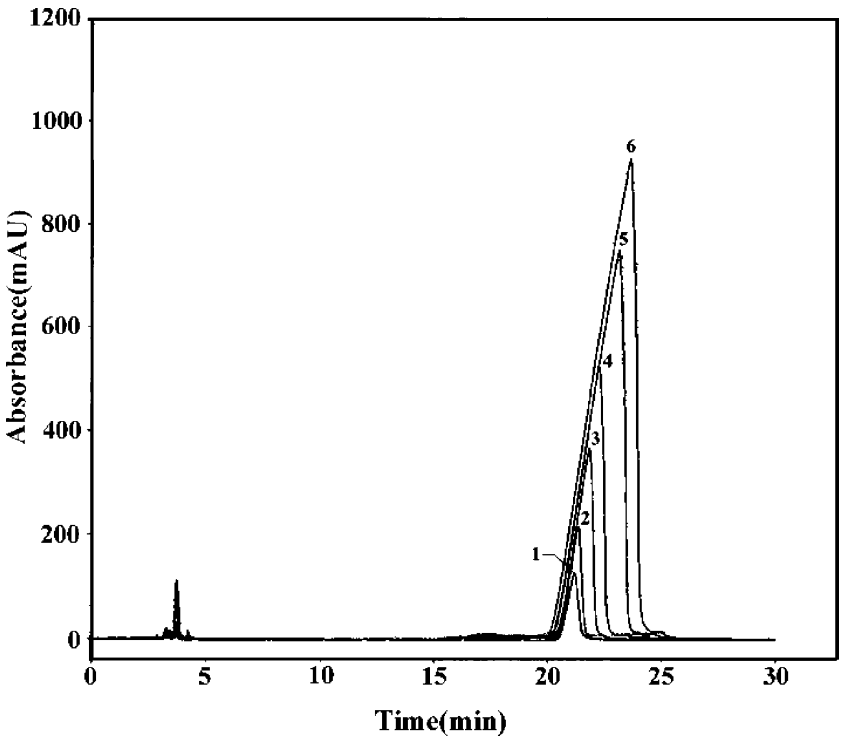

Fig. 8. Chromatograms of epirubicin of increasing sample sizes. Mobile phase: sodium formate buffer $(20 \mathrm{mM}, \mathrm{pH} 2.9)$-acetonitrile $(10: 90, \mathrm{v} / \mathrm{v})$. Sample amounts injected are: (1) $0.8 \mu \mathrm{g}$; (2) $4.1 \mu \mathrm{g}$; (3) $8.2 \mu \mathrm{g}$; (4) $16.4 \mu \mathrm{g}$; (5) $32.2 \mu \mathrm{g}$; (6) $49 \mu \mathrm{g}$.

accounted for in terms of irreversible adsorption. As easily inferred from these results that the occurrence of irreversible adsorption is strongly dependent on the eluent $\mathrm{pH}$.

Additionally, when the ionic strength is increased by addition of $\mathrm{NaNO}_{3}$ in the neural or weakly acidic mobile phases, there is no noticeable improvement in irreversible adsorption observed intuitively, but it causes competing effects on adsorption and stability, resulting in a reduction of the retention time. Also, the irreversible adsorptions of these compounds can be reduced by increasing water content or by substituting an equal concentration of methanol for acetonitrile in these $\mathrm{pH}$ conditions.

\subsection{Effect of sample size}

The retention dependence on sample size of epirubicin and its analogues was investigated at low buffer $\mathrm{pH}$ where satisfactory resolution and reproducibility were achieved. Fig. 8 shows the typical chromatograms of epirubicin of increasing sizes $(0.8-49 \mu \mathrm{g})$ on silica with formate buffer $(20 \mathrm{mM}$, $\mathrm{pH} 2.9)$-acetonitrile (10:90, v/v) as mobile phase. As can be seen from Fig. 8, the elution profiles have shown leading peaks, with a diffuse front and a steep rear, and the retention time of the peak increases with increasing sample size. These profiles are similar to the classical profile observed for a component having an anti-Langmuirian isotherm [28], and just opposite to the peaks with a sharp front and a tailing rear corresponding to a Langmuirian isotherm at the overloading conditions in RPLC [21]. Anti-Langmuir behavior of a component in sample mixture displays a negative displacement effect in competitive adsorption on the surface of adsorbent, leading to a disturbance of band separation in nonlinear preparative scale [28]. The conditions causing
Table 2

Effect of sample sizes on retention factor $\left(k^{\prime}\right)$, column efficiency $\left(N / N_{0}\right)$ and asymmetry factor $\left(A_{\mathrm{s}}\right)$ for epirubicin and its analogues on silica with sodium formate buffer $(20 \mathrm{mM}, \mathrm{pH} 2.9)$-acetonitrile $(10: 90, \mathrm{v} / \mathrm{v})$ as the mobile phase

\begin{tabular}{lclll}
\hline Compound & Sample size $(\mu \mathrm{g})$ & $k^{\prime}$ & $N / N_{0}$ & $A_{\mathrm{s}}$ \\
\hline Epirubicin & 0.25 & 5.69 & 1 & 1.08 \\
& 0.5 & 5.70 & 0.97 & 1.10 \\
& 0.8 & 6.07 & 0.71 & 0.80 \\
& 4.1 & 6.12 & 0.55 & 0.70 \\
& 8.2 & 6.28 & 0.26 & 0.64 \\
& 16.4 & 6.42 & 0.14 & 0.61 \\
& 32.2 & 6.70 & 0.08 & 0.59 \\
& 49 & 6.88 & 0.05 & 0.57 \\
& 0.25 & 4.50 & 1 & 1.02 \\
& 0.7 & 4.52 & 0.95 & 1.00 \\
& 2.4 & 4.85 & 0.78 & 0.89 \\
& 4.7 & 4.95 & 0.65 & 0.77 \\
Daunorubicin & 9.4 & 5.00 & 0.47 & 0.68 \\
& 18.8 & 5.16 & 0.27 & 0.62 \\
& 0.25 & 5.27 & 1 & 0.99 \\
& 0.7 & 5.31 & 0.96 & 1.00 \\
& 2.4 & 5.69 & 0.74 & 0.84 \\
& 4.7 & 5.80 & 0.64 & 0.74 \\
Doxorubicin & 0.25 & 6.73 & 1 & 1.03 \\
& 0.5 & 6.74 & 0.97 & 1.06 \\
& 0.8 & 7.31 & 0.72 & 0.79 \\
& 4.4 & 7.49 & 0.50 & 0.68 \\
& 8.8 & 7.64 & 0.29 & 0.62 \\
& 17.6 & 8.04 & 0.16 & 0.58 \\
& & & &
\end{tabular}

a component having an anti-Langmuir behavior should be avoided or changed in order to convert the behavior from anti-Langmuir to Langmuir type. Epidaunorubicin, daunorubicin and doxorubicin give similar chromatographic behavior to epirubicin with increasing sample size, such as retention factor $\left(k^{\prime}\right)$, column efficiency (described as $N / N_{0}$, plate number divided by the maximum plate number) and peak asymmetry $\left(A_{\mathrm{s}}\right)$ as shown in Table 2 .

\section{Applications}

Fig. 9 shows HILIC mode chromatogram of epirubicin in its raw product. The resolution between the peaks corresponding to impurity daunorubicin and epirubicin is $>2.0$. Compared to RPLC, the normal phase retention behavior of HILIC offers the potential for dramatic changes in selectivity with quite simple mobile phase. The compositions of the eluents used in most RPLC methods are comparatively complex [15-20], and phosphate buffers as well as anionic surfactants are favored in HPLC-UV but not in MS detection. It can be expected that HILIC on silica with high-organic low-ionic-strength volatile buffer will be a good choice for developing LC-MS assay for quantifications of epirubicin and its metabolites in biological matrices. Also, because of higher organic content of the mobile phase in HILIC than in RPLC, lower pressure drop is usually observed on the 


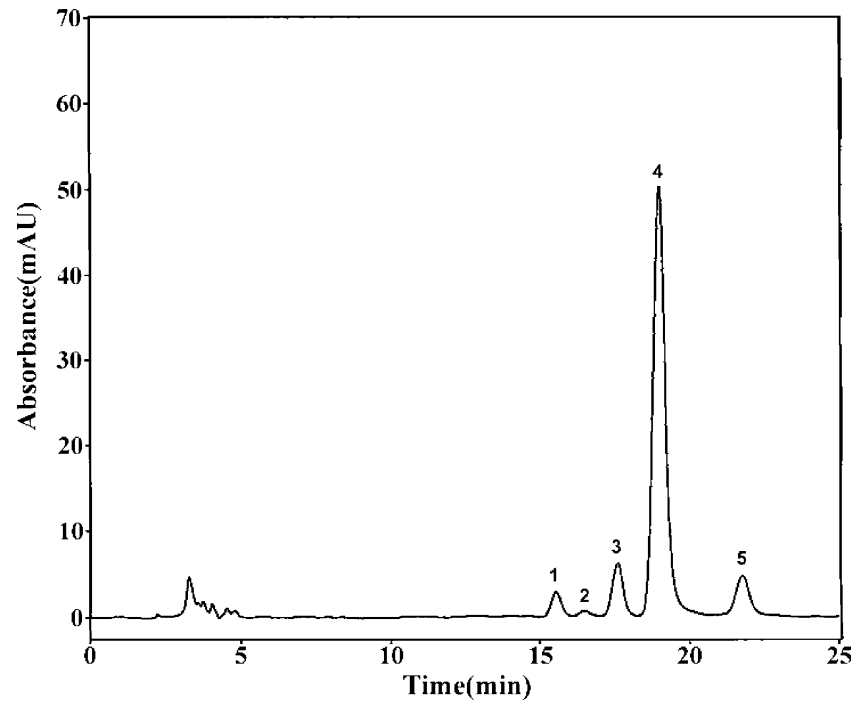

Fig. 9. Chromatogram of the analysis of epirubicin hydrochloride in a raw material. Conditions: Kromasil KR100-5SIL (5 $\mu \mathrm{m})$; mobile phase: sodium formate buffer $(30 \mathrm{mM}, \mathrm{pH} 2.9)$-acetonitrile $(10: 90$, v/v). Peaks: (1) epidaunorubicin, (2) unknown compound, (3) daunorubicin, (4) epirubicin, (5) doxorubicin.

silica column. Moreover, unlike classical NPLC, where the trace amount of water in the mobile phase has to be strictly controlled [29], the mobile phase in HILIC can be easily prepared.

\section{Conclusions}

HILIC using high-purity silica column with an aqueousorganic eluent has been shown to be a very powerful technique for separating epirubicin and its analogues. The degree of retention and the selectivity of this chromatographic system are largely dependent on the nature and composition of the organic modifier of the eluent, the $\mathrm{pH}$ of the buffer and the ionic strength in mobile phase. Especially, the choice of organic modifier is essential for successful separation of these analogues. According to our results, acetonitrile provides optimum separation, selectivity and efficiency compared to methanol, isopropanol and THF.

Our studies on the effect of organic modifier, buffer $\mathrm{pH}$ and ion strength well support that the retention mechanism of the four analogues is a combination of adsorption and ion exchange under HILIC conditions. The variation between the two contributions causes a change of the elution order. Although the satisfactory resolutions have been achieved both in acidic and neutral buffered mobile phases, however, the irreversible adsorption of the four solutes on silica column takes place under weakly acidic or neutral conditions, which can be reduced by changing the eluent conditions.
Finally, when increasing sample sizes in acidic mobile phase, the peak profiles of epirubicin and its analogues have a slanted front and a sharp tail, associated with a solute having an anti-Langmuir isotherm under column overloading condition. The proposed HILIC method not only provides a useful method to the pharmaceutical industry in the determination of epirubicin and its impurities, but also offers the potential to match LC-MS for assay of epirubicin and its metabolites.

\section{References}

[1] J. Layne, J. Chromatogr. A 957 (2002) 149.

[2] L.K. House, J. Ramirez, M.J. Ratain, J. Chromatogr. B 720 (1998) 245.

[3] J.M. Roberts, A.R. Diaz, D.T. Fortin, J.M. Friedle, S.D. Piper, Anal. Chem. 74 (2002) 4927.

[4] B.A. Olsen, J. Chromatogr. A 913 (2001) 113.

[5] A.J. Alpert, J. Chromatogr. 499 (1990) 177.

[6] H. Schlichtherle-Cerny, M. Affolter, C. Cerny, Anal. Chem. 75 (2003) 2349.

[7] H. Tanaka, X. Zhou, O. Masayoshi, J. Chromatogr. A 987 (2003) 119.

[8] M.A. Strege, S. Stevenson, S.M. Lawrence, Anal. Chem. 72 (2000) 4629.

[9] M.S.S. Curren, J.W. King, J. Chromatogr. A 954 (2002) 41.

[10] B.M. Lampert, J.T. Stewart, J. Chromatogr. 495 (1989) 153.

[11] B. Law, Trends Anal. Chem. 9 (1990) 31.

[12] J. Nawrocki, J. Chromatogr. A 779 (1997) 29.

[13] G. Zagotto, B. Gatto, S. Moro, C. Sissi, M. Palumbo, J. Chromatogr. B 764 (2001) 161.

[14] F. Arcamone, Doxorubicin. Academic Press, New York, 1981.

[15] European Pharmacopoeia, European Directorate for the Quality of Medicines, Council of Europe, fourth ed., Strasbourg, 2002, p. 1109.

[16] G. Nicholls, B.J. Clark, J.E. Brown, J. Pharm. Biomed. Anal. 10 (1992) 949.

[17] A. Rudolphi, S. Vielhauer, K.S. Boos, D. Seidel, I.M. Bathge, H. Berger, J. Pharm. Biomed. Anal. 13 (1995) 615.

[18] I.K. Barker, S.M. Crawford, A.F. Fell, J. Chromatogr. B 681 (1996) 323.

[19] R. Ricciarello, S. Pichini, R. Pacifici, I. Altieri, M. Pellegrini, A. Fattorossi, P. Zuccaro, J. Chromatogr. B 707 (1998) 219.

[20] A.U. Kulikov, L.P. Loginova, L.V. Samokhina, Chromatographia 57 (2003) 463

[21] Y. Qi, J.X. Huang, J. Liq. Chromatogr. Rel. Technol. 24 (2001) 2837.

[22] Y. Qi, J.X. Huang, J. Chromatogr. A 959 (2002) 85.

[23] V.R. Meyer, Practical High-Performance Liquid Chromatography, Wiley, Chichester, 1999, p. 70.

[24] R.J.M. Vervoort, E. Ruyter, A.J.J. Debets, H.A. Claessens, C.A. Cramers, G.J. de Jong, J. Chromatogr. A 931 (2001) 67.

[25] S. Espinosa, E. Bosch, M. Rosés, Anal. Chem. 74 (2002) 3809.

[26] L.R. Snyder, J. Chromatogr. 255 (1983) 3.

[27] R.P.W. Scott, Adv. Chromatogr. 20 (1982) 169.

[28] G. Guiochon, S.G. Shirazi, A.M. Katti, Fundamentals of Preparative and Nonlinear Chromatography, Academic Press, Boston, 1994.

[29] J.J. Kirkland, C.H. Dilks Jr., J.J. DeStefano, J. Chromatogr. 635 (1993) 19. 